\title{
Corrosion of Metal Films Observed Using In Situ and Ex Situ Electron Microscopy
}

See Wee Chee ${ }^{1}$, Frances M. Ross ${ }^{2}$, David Duquette ${ }^{1}$ and Robert Hull ${ }^{1}$

1. Department of Materials Science and Engineering, Rensselaer Polytechnic Institute, 110 8th Street, Troy, NY 12180, USA.

2. IBM T. J. Watson Research Center, 1101 Kitchawan Road, Yorktown Heights, New York 10598, USA.

Localized corrosion refers to the accelerated dissolution of metals and alloys due to the breakdown of their protective passive films at select sites [1]. Developing an understanding of localized corrosion phenomena has significant practical importance due to the deleterious effects of pit formation on the integrity of structural materials. However, localized corrosion is a challenging problem to study because of (1) the small dimensions of pit initiation sites; (2) the short timeframe between pit initiation and pit propagation; (3) the scarcity and stochastic nature of pitting events; (4) the difficulty of characterizing the thin passive film in aqueous environments; and (5) the dynamic evolution of the surface, potential and solution concentration gradients with time [2]. Here, we combine in situ liquid flow cell electron microscopy and ex situ electron microscopy to examine localized corrosion events in thin metal films.

For the experiments, 100nm thick Al films were deposited on the entire surface of a microchip that forms half of the liquid flow cell using electron beam evaporation at a rate of $\sim 2 \AA$ per s. The cell was placed in a Hummingbird Scientific liquid flow holder and examined in a $200 \mathrm{kV}$ JEOL JEM-2011 LaB 6 transmission electron microscope (TEM). Images were collected with a beam current of 4-6 mA per $\mathrm{cm}^{2}$. $\mathrm{NaCl}$ solutions of different concentrations were delivered from outside the TEM using a syringe pump. Due to the long duration of these experiments, the images were taken at intervals in a snapshot manner to minimize the electron dose on the sample. To mitigate the resolution loss due to imaging through liquid, the liquid was selectively de-wetted from the cell using a mixture of water and ethanol, a procedure that does not require focusing of the beam (see [3] for details). Post-mortem ex situ studies were performed using optical microscopy and scanning electron microscopy, in addition to TEM.

By changing the concentration of $\mathrm{NaCl}$ from $0.01 \mathrm{M}$ to $1.0 \mathrm{M}$, different features (Figure 1) were observed in the $\mathrm{Al}$ films [3]. In the $0.01 \mathrm{M} \mathrm{NaCl}$ solution, blisters were found in the film after 15 hours. In the $0.1 \mathrm{M}$ $\mathrm{NaCl}$ solution, we observed anomalous crystalline structures that were 2-3 times the size of the deposited $\mathrm{Al}$ grains. In the 1.0M NaCl solution, fractal corrosion structures were observed over the entire film. We will discuss the relationship between these features and models for pitting corrosion.

The introduction of a second metal is known to greatly affect corrosion behaviour [1]. The Al films were implanted with $\mathrm{Au}+$ ions using a mass-filtered focused ion beam in an array consisting of single spots separated by 5 microns (see [4] for details of the FIB setup). The dose was $\sim 10^{7}$ ions per spot which corresponds nominally to $0.03 \mathrm{at} \% \mathrm{Au}$ in $\mathrm{Al}$ (estimated by assuming that the ions are evenly distributed over the entire surface and to a range of $19 \mathrm{~nm}$ as calculated with SRIM, however, note that Au was implanted as focused spots in the experiment). Since $\mathrm{Au}$ is the more noble metal in the electrochemical series, Al is expected to preferentially corrode. Figure 2 shows post-mortem images recorded in the optical microscope, SEM and TEM after 3 hours immersion in $0.1 \mathrm{M} \mathrm{NaCl}$ solution (not in the liquid TEM holder). The implanted areas show significantly less pitting compared to the surrounding areas. We will discuss the effect of the implantation in changing both crystal structure and composition, and consider the 
opportunities for liquid cell electron microscopy to provide quantitative measurements of localized corrosion phenomena in films of varying composition.

\section{References:}

[1] E. McCafferty, "Introduction to Corrosion Science”, (Springer, New York)

[2] G.S Frankel, Journal of the Electrochemical Society, 145, (1998), p.2186, G.S. Frankel and N.

Sridhar, Materials Today, 11 (2008) p. 39.

[3] Chee et al, MRS Proceedings, 1525, p. mrsf12-1525-ss11-03, Chee et al, Microscopy and

Microanalysis (2014), accepted.

[4] Chee et al, Ultramicroscopy, 127 (2013) p. 126.

[5] J. Ziegler, "Particle Interactions with Matter", (www.srim.org)

[6] The authors acknowledge funding from the NYSTAR Focus Center, Grant \# C080117 and Mr. Prabhu Balasubramanian for his help in the film deposition.
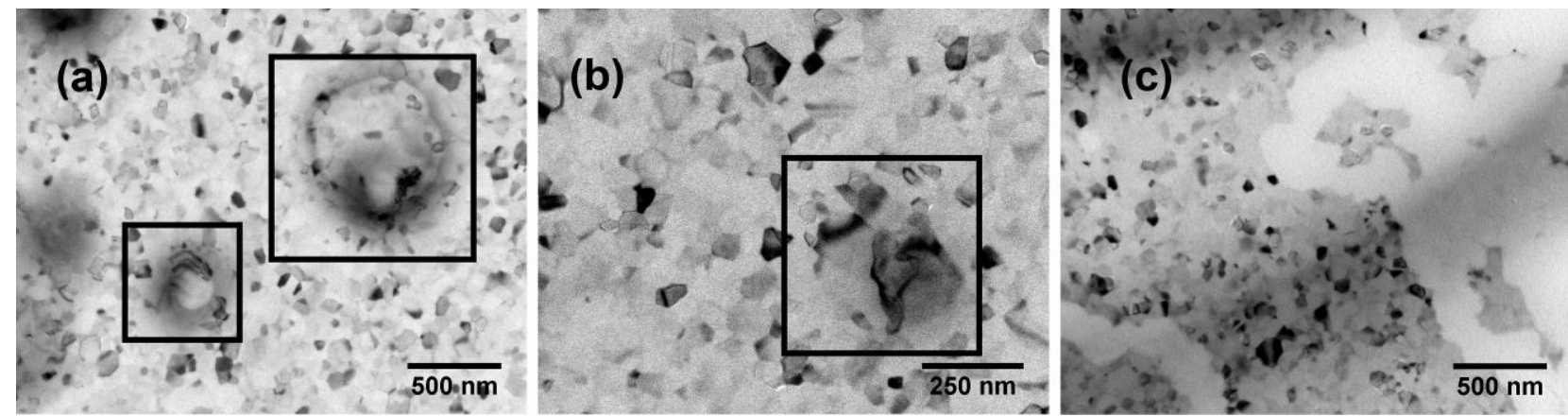

Figure 1. Corrosion structures in $\mathrm{Al}$ thin films immersed in $\mathrm{NaCl}$ solutions as observed using liquid cell transmission electron microscopy. The as-deposited films are polycrystalline with 100nm grain size. (a) Blisters in $0.01 \mathrm{M} \mathrm{NaCl}$ after $15 \mathrm{hrs}$ (highlighted in black square), (b) anomalous structures in $0.1 \mathrm{M} \mathrm{NaCl}$ after 5 hours (highlight in black square) and (c) Fractal corrosion patterns in $1.0 \mathrm{NaCl}$ after 4 hours (white tracks)
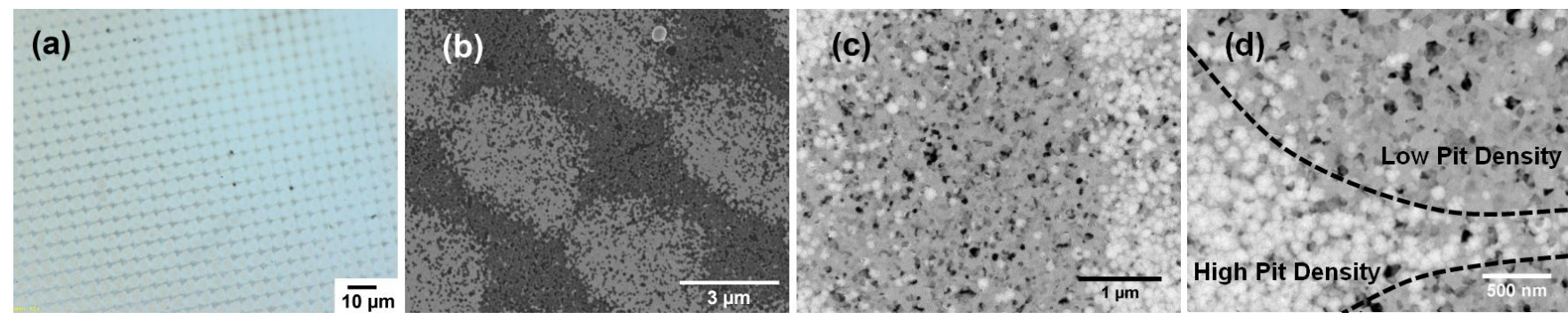

Figure 2. Post-mortem images taken with (a) optical microscope, (b) scanning electron microscope $(10 \mathrm{kV}$ accelerating voltage) and (c) transmission electron microscope of an Al film that has been implanted with focused $30 \mathrm{kV} \mathrm{Au}{ }^{+}$ions in an array. The sample has been immersed for 3 hours in $0.1 \mathrm{M}$ $\mathrm{NaCl}$ solution (not in the liquid TEM holder). It can be seen from (a) and (b) that the implanted pattern is re-produced in the Al film where the darker regions are where Al has been corroded away. Image (c) shows that the density of pits (white spots) within and around the implanted regions is significantly less that the surrounding area, $(d)$ is a higher magnification TEM image where the high pit density regions and low pit density regions are demarcated with a dotted line. 